\title{
Limits of Truth: Exploring Epistemological Approaches to Argumentation
}

\section{Michael H.G. Hoffmann Georgia Institute of Technology}

\begin{abstract}
Some proponents of epistemological approaches to argumentation (Biro, Siegel, Lumer, Goldman) assume that it should be possible to develop non-relative criteria of argument evaluation. By contrast, this paper argues that any evaluation of an argument depends (a) on the cognitive situation of the evaluator, (b) on background knowledge that is available for this evaluator in a certain situation, and (c) - in some cases-on the belief-value-system this person shares.
\end{abstract}

\begin{abstract}
Résumé: Les partisans de l'approche épistémologique (Biro, Siegel, Lumer, Goldman) supposent qu'il devrait être possible de développer des critères nonrelatifs pour évaluer des arguments. Par contraste, je soutiens que n' importe quelle évaluation d'un argument dépend (a) de la situation cognitive de l'évaluateur, et (b) des connaissances générales qui lui sont accessibles pour cette évaluation, et (c) dans certains cas, du système de valeurs de cette personne.
\end{abstract}

Keywords: argument evaluation and quality, standards of argumentation, epistemological approaches, relativism, objectivism, conflict, disagreement, cognitive situation, backgrouind knowledge, belief-value-system

\section{Introduction}

So-called "epistemological approaches to argumentation" focus on the idea that the main function of arguments and argumentations is to generate knowledge or justified belief. The primary goal is not to find consensus between participants or to persuade somebody to believe in a certain claim, but to produce rationally "justified" beliefs or, as others say, "true beliefs" (Biro \& Siegel 1992; Feldman 1994, 176; Goldman 1999; Lumer 1990, 30; 1991; Siegel \& Biro 1997, 278). Based on this, some proponents of an epistemological approach assume that the quality of arguments and argumentations can be evaluated by more objective criteria than, say, by observing a group of people's reactions to an argument or by performing a survey that shows how "successful" a certain argument is.

The question I want to discuss in this paper is whether this is possible. Is it convincing, as many claim, that there are non-relative standards of argument evaluation? Are the standards the literature provides sufficient to justify in each case a clear distinction between "good" and "bad" arguments? Since the answer to both these questions seems to be connected with the possibility that arguments can generate true beliefs, or knowledge, the broader focus of this paper will be on limits of truth in argumentations. 
Looking for limits means, of course, that I am less interested in things that are not really controversial. There is no question that we have to teach certain standards of argument construction and evaluation in order to improve the practice of argumentation. But the question is: is it possible to formulate standards of argument evaluation in a way that an evaluation's result is solely determined by the standards applied, so that whoever used these criteria to evaluate an argument would come to the same result? Can those standards be applied to each and every argument in a way that a clear and unquestionable decision regarding its quality is possible? If that would be possible, many other things would be possible too. For example, it would be possible based on those "objective" standards to decide objectively which party in a conflict on a certain issue has the better argument since, at the end, there can only be one truth.

Formulating the question in this way would presuppose a very strong version of an epistemological approach to argumentation: a unique truth and objective criteria to decide whether an argument for a proposition claimed to be true is a good one or a bad one. By contrast, a weaker--and relativist-version might accept the possibility of true propositions but could claim, for example, that evaluation standards depend on a community of people accepting these standards in a certain historical, social, cultural, cognitive, emotional, etc., situation. If a theory rejects the possibility of truth altogether, I would not call it an "epistemological" approach. There is, of course, a broad area of intermediate possibilities between both these extreme versions of epistemological approaches to argumentation. Questioning the "limits" of truth means a critical reflection on where to put exactly the boundary between what should be acceptable and what is not.

Any discussion of these possibilities depends, of course, on how the concepts of "truth", "objectivity", and "relativity" are defined. In order to cope with this problem, I will start with what has been provided by the literature so far. The strategy is to discuss a range of epistemological approaches in a way that the authors' terminology can be critically reflected as well. With regard to the concepts of "argument" and "argumentation," I should say in advance that I am following Alvin Goldman in using "argument" for a "set of statements or propositions schematized as ' $R_{1}, \ldots, R_{\mu}$, therefore $P$ ' ... It contains one or more premises and a conclusion, where the premises jointly provide evidential support (not necessarily conclusive) for the conclusion". "Argumentation", on the other hand, is used if "a speaker presents an argument to an audience, in which he asserts and defends the conclusion by appeal to the premises". It is "monological argumentation" when there is only one speaker, and a "dialogical" when there are two or more arguing for different sides (Goldman 1999, 131). Thus, the main difference between an "argument" and an "argumentation" is that if we focus on the former, it is sufficient to reflect on the set of statements that form an argument, while if we focus on the latter, we have to take the context into account in which this set of propositions is formulated. 


\section{Biro and Siegel's "intrinsic" quality of arguments}

The strongest version of an epistemological approach to argumentation, as far as I can see, has been developed by John Biro and Harvey Siegel. They claim that it is possible to determine "the goodness of good arguments" based on what they call "an internal account" (Biro \& Siegel 1992, 86). The idea seems to be that arguments themselves possess "certain epistemic properties, such as epistemic seriousness", and if those properties are missing, we get a bad argument. It is, however, hard to understand what exactly the authors intend to say: "the hallmark of a good argument is obviously to provide an intrinsic explanation of what is wrong when an argument lacks such a property" (ibid., 93). Is an "intrinsic explanation" the same as an "internal property" of an argument? What does it mean that an argument "provides" an explanation? Does it mean that any reader of such an argument would automatically generate such an "explanation"? The "epistemic property" seems to be connected somehow with providing "a bridge from known truths or justified beliefs to as yet unknown (or at least unrecognized) truths or as yet unjustified beliefs. An argument aims at, and a good one succeeds in, leading an inquirer or an audience from some proposition(s) whose truth or justifiedness they accept to others whose truth or justifiedness they will see themselves as having good reasons to accept on its basis" (Biro \& Siegel 1992, 92, their emphasis).

But how can it be possible that all this depends on the argument itself (cf. Siegel 1999,184$)$ ? Is there any 'knowledge generating power' that is present in some arguments but not in others? The remaining part of their article confirms a certain vagueness of this approach:

What is it for a conclusion to be warranted by an argument? The short answer is: a conclusion is warranted by an argument if the argument renders belief in the conclusion rational, if the premises of the argument provide good reasons for and so warrant its conclusion. ... When does an argument render a conclusion rationally justified; belief in its conclusion (on its basis) rational? Here we enter into contentious epistemological territory and must proceed with caution. In general, an argument renders belief in a claim (on its basis) rational when the reasons provided by the argument for the claim meet criteria concerning the goodness of reasons. ... When are reasons 'good enough'? Here we reach the heart of epistemology ... There are a wide variety of principles of reason assessment, ranging from the subject-neutral principles of logic to the subject-specific principles governing reason assessment in the special fields. ... Moreover, the diverse epistemological stances available to the argumentation theorist will yield substantively different principles of reason assessment and, with them, different criteria of argument evaluation. (Biro \& Siegel 1992, 97-99)

What started impressively strong with the claim that there are "epistemic properties" of arguments "themselves" that allow their evaluation independently of any relation to anything else ends so finally in an unspecified variety of "different principles" that range from "subject-neutral" ones like logical validity to "subject-specific" 
ones. However, since Biro and Siegel do not specify their "principles of reason assessment", there is no way to assess their approach. Their claim that good arguments have certain "epistemic properties" remains vague.

In a more recent article, Harvey Siegel defended the thesis "that the quality of arguments is a feature of the argument itself" by criticizing a so-called "multiculturalist approach to argument quality" (Siegel 1999, 184). He tries to demonstrate the weakness of four arguments that proponents of culture- and perspective-relativity might formulate.

In his first argument, Siegel accepts that there is no "perspectiveless perspective", no "cosmic exile", and no vantage point beyond any "conceptual scheme" (191). But, he says, we are not "trapped" forever in any specific perspective; we are able to learn and, thus, to transcend any perspective. However, it is hard to imagine that a relativist would deny the possibility of developing and transcending perspectives, and of shifting between different perspectives. This first argument poses rather a problem for Siegel himself. It is obviously contradictory to claim, on the one hand, "that we inevitably judge from some framework or other" (191) and, on the other, that "the quality of the argument is a feature of the argument itself" (184). If we can determine the quality of an argument only by means of a judgment, and if all judgments are determined by the evaluator's framework, we have to conclude that any argument evaluation is relative to those frameworks and not a question of "the argument itself".

Problematic is also Siegel's second argument. He says that we

are not denying that our principles of argument evaluation and criteria of argument quality are local and particular, in the sense that they are ours: articulated and endorsed by us, in our particular historical/cultural context. But acknowledging their particularly [sic] does not preclude us from proclaiming their universality: that is, their legitimate applicability to arguments, considered independently of their location. (193)

It might indeed be possible that the evaluation standards I am using in my particular situation happen to be "universal" standards, but how could I know that? And how could it be possible for anyone to justify the claim that her or his standards are in fact "the" universal standards?

However, the "main difficulty with conceiving of argument quality in culturally relative terms" is, according to Siegel, the following. He maintains that "any advocate of a culturally relative conception of argument quality" who claims rationality for his or her arguments - that is, that these arguments are "warranted by the reasons offered in its support"- "must presuppose" just those absolute standards she is trying to get rid of (194). This argument is similar to a variety of arguments Siegel developed already in his book Relativism Refuted in order to demonstrate that relativism as an epistemological position is "self-defeating and incoherent" (Siegel 1987). If the relativist Protagoras in Plato's Theaetetus claims "to be right" he presupposes what he rejects (4). If he claims that all opinions are true only for 
those who believe them, then his own claim is at the same time true and false because he believes it to be true while his opponents think it is false (5f.), etc. All these arguments, however, are simply confusing what a relativist might claim and what Siegel himself is not ready to give up: a presupposed non-relative understanding of "rationality", "rightness", "truth", and so forth. An extreme relativist, however, would not have any problems to claim-as Protagoras does - that his statement is only true for himself; he does not care whether it is false for others. And a moderate relativist could hint at the difference between her hope to argue rationally and to say the truth and her inability to prove that she is right. In a way, the whole debate is about who is supposed to carry the burden of proof. Siegel is right when he says that a relativist who claims "the truth" of relativism, or who tries to "justify" relativism in a non-relative way, would contradict himself. But why should he or she try to do that? From my point of view, relativism is not a "theory", but an assumption that is inevitable if we do not succeed in proving the possibility of nonrelative knowledge.

Siegel's fourth and last argument against relativism in argumentation theory attacks an observation that has been an important starting point especially for "postmodern" thinkers (cf. Lyotard $1984<1979>$, 1993; Vattimo $1991<1985>$ ), but earlier also for the Frankfurt School (Horkheimer \& Adorno $2002<1947>$ ) and later for feminist epistemologists (cf. Smith 1990; Longino $1996<1993>$; Harding $1996<1993>$ ). It is the observation that distinguishing certain arguments based on their "rationality" equals an "exercise of power" (Siegel 1999, 197). Thus, an "impersonal, transcultural view of argument quality" could be criticized as a "rhetorical, power-laden construct" (197); a trick we use to get an advantage in all these political power games. Siegel's main objection against this view is that it "plagues culture-relative as well as transcultural views of argument quality" (ibid.). But this underestimates the fact that it makes a difference whether people are claiming to possess the better argument only because they belong to a certain culture or because they are following a universal standard of correct thinking. The critics of rationality as a universal standard always emphasized that it is more dangerous when "exercises of power" can be hidden behind claims on universality, and that universality itself is a totalitarian concept.

However, the problem of power in discourse can also be viewed from the opposite direction. George Boger has recently argued that relativist approaches that are replacing the "truth requirement of a good argument" by "an acceptability requirement" might—given "widely divergent audiences not sharing a common interest but engaged in an 'argumentative conversation" - "unfairly favor the stronger of the 'disputants' and place the weaker at a decided disadvantage" (Boger 2005,189 ). Thus, he is arguing exactly in the opposite direction: it is not that truth orientation is a means to establish power, but not focusing on truth means, first of all, that we leave a space unoccupied that finally will be structured only by power. This is indeed a good point. It might seem that a relativist approach justifies at the 
end everything-implicitly, at least-if truth is no longer an absolute standard to control the rationality of human communication. But what are we supposed to do if - as in many cases-we simply do not have access to "the" truth? We cannot simply "secure objective knowledge" (189) if objective knowledge is beyond what we can achieve by our means.

Taking Boger's objection seriously should lead to a form of relativism, I would argue, that does not abandon the notion of truth altogether. Following the argument developed by Bernard Williams in Truth and Truthfulness, we should save truth at least as a "value", that is as an ethical standard. Even if it is impossible to formulate a final and complete justification of truth claims, we can respect truth as an ideal that ought to be both the goal of our endeavors and a behavioral guideline that governs the practice of argumentation. In this latter sense, Bernard Williams claims "Accuracy and Sincerity" as the "basic virtues of truth":

$[Y]$ ou do the best you can to acquire true beliefs, and what you say reveals what you believe. The authority of academics must be rooted in their truthfulness in both these respects: they take care, and they do not lie. (Williams 2002, 11)

Truth should be viewed, first of all, as a moral value that requires accuracy, sincerity, seriousness, and open-mindedness in our attempts to understand.

We should mention at this point that also Siegel, after refuting relativism, formulates an understanding of what he calls an "absolutist epistemology" that allows a belief system to be "both self-correcting and corrigible" (Siegel 1987, 161). The sort of "absolutism" he favors requires "the possibility of objective, non-question-begging evaluation of putative knowledge claims, in terms of criteria which are taken as absolute but which nonetheless admit criticism and improvement" (162). This way, however, "objective, non-question-begging" evaluation criteria are not "intrinsic properties" of an "argument itself", but simply presupposed by individuals ("taken as"). But is there any justification for such a presupposition beyond the subjective framework of a certain person?

Siegel claims that his "fallibilist, non-dogmatic, self-correcting absolutism, which provides for objectivity in the evaluation of knowledge claims and the possibility of criticism and improvement of criteria which guide such evaluation, is entirely compatible with the rejection of relativism" (162). I find it hard to see this compatibility. Only if we turn the meaning of concepts like "objectivity" and "absolute" upside down would it be compatible. Remember that the Latin absolutus (from ab-solvo) means literally "detached, uncoupled", and "objective" in philosophy is usually opposed to "subjective". Both terms emphasize independence from any individual perspective and activity. However, if we presuppose such an independence of evaluation criteria, how can we change them without making them relative to what we are doing with them? Relative "to time, to place, to society, to culture, to historical epoch, to conceptual framework, to personal training or conviction"as Siegel introduces "relativism" in his book (xiii)? 


\section{Lumer's principles of argument evaluation}

While Biro and Siegel do not specify evaluation criteria beyond the rather vague claim that there "are a wide variety of principles of reason assessment", and while it remains unclear how Siegel can reconcile the "objectivity" of those criteria with the subjective dimension of criteria improvement, Christoph Lumer, on the one hand, offers a highly elaborated distinction of "epistemological principles ... which formulate sufficient conditions for the truth or acceptability of propositions" (1991, 101 ) and, on the other hand, provides some ideas that might be helpful for clarifying the problem of relativity and non-relativity of argument evaluation (1991; cf. also 1990).

Lumer's starting point is the consideration that it would be unsatisfactory-at least from a philosophical point of view -if the fact that validity criteria as used in deductive logic cannot be applied to evaluating all kinds of arguments would lead to a situation in which the quality of arguments can only be determined by "finding out whether a certain type of speech would convince some addresses" $(1991,98)$. His goal is to define "validity criteria" for argumentations, whereby "validity" is defined here- a bit unusually — as fulfilling an argumentation's "standard-function" (100). These criteria are at the same time criteria for the assessment of arguments and argumentations. (Note that Lumer's definition of "argumentation" corresponds to our "argument", while his "argument" is what I am calling "reason").

According to Lumer, the "standard-function of argumentations" is "to convince rationally or to show the acceptability of the thesis" (100), that is to generate knowledge, or to lead the argument's addressee "to the truth" $(1990,6,43 \mathrm{ff}$.). The essential idea of his approach is to use this standard-function "for assessing the usefulness of proposed criteria for the validity of argumentations" $(1991,100)$. The standard-function, obviously, can be fulfilled by quite different meansdepending on what kind of argument we use: knowledge can be generated, for example, by deduction (in this case we use "conclusive" principles of validity) or by "revelation" if we think that everything "is true what has been revealed in the Bible" (101). In between both these extremes, we should find what an epistemological approach to argumentation is all about: "efficient" principles; that is, principles which are not conclusive but "efficient in the sense that their correct use only. leads to acceptable beliefs; these are probably true or verisimil beliefs" (101). Inductive principles, for example, are efficient but not conclusive. One of Lumer's goals is to define those "efficient principles".

In his book Praktische Argumentationstheorie, Lumer develops those efficient principles by a method called "idealizing hermeneutics" (1990, $10 \mathrm{ff}$; all translations from this book are mine). The goal of this method is to define the standard function of arguments based on an empirical analysis of those arguments that seem to be valid and adequate, to determine the rules (or principles, or conditions) that are necessary to fulfill this standard function, and finally to structure these rules "in axiomatic form" (13). 
An example for the outcome of this procedure is provided in Lumer (1991) with regard to validity criteria for "ideal deductive argumentations" (102 ff.). Most of these criteria refer to the argument itself. They concern the identification of an argument's structure, the "guarantee of truth" ("The propositions of the arguments of a complete and valid deductive argumentation are true and they deductively imply the proposition of the thesis"), and what Lumer calls "adequacy in principle: ... there must be at least one person sometime who knows that the arguments are acceptable, but who doesn't know this about the thesis" (102). This condition is necessary since "an instrument is an instrument" only if its function can be fulfilled in principle.

However, there is also one "validity principle" that refers to an argument's addressee. It is called "Adequacy in concrete situations":

A valid deductive argumentation will be adequate for rationally convincing an addressee of the thesis if the following conditions are fulfilled: 1. The addressee is linguistically proficient, open-minded, attentive, and discriminating; 2 . he knows that the arguments are acceptable but doesn't know this about the thesis; 3 . the relation of implication between the propositions of the arguments and the proposition of the thesis is sufficiently direct so that it can easily be grasped by the addressee; if necessary, for fulfilling this condition the argumentation will have to be divided in a few subargumentations; 4 . the set of arguments contains only arguments which are necessary for the deduction of the thesis. (Lumer 1991, 102)

Based on this principle, an argument evaluation has to go beyond analyzing the argument itself. To determine whether an argument is good or bad, we have also to check whether the four conditions listed in the quote are fulfilled in a concrete situation. If the standard function of an argument is to generate knowledge by leading somebody to the truth, then not only the argument itself must fulfill certain conditions but also the argument's addressee. The quality of an argumentation depends therefore also.on what can be called the "cognitive situation" of an argument's addressee.

If the quality of an argumentation depends on the addressee's cognitive situation, one might suspect a form of relativism here. However, there is - at least according to Lumer's presentation of this point-no relativity in the evaluation of an argumentation. Any evaluator should be able to look at a concrete situation and to tell whether the cognitive conditions formulated in the quote are fulfilled or not. If they are fulfilled, as well as the criteria concerning the argument itself, then the argumentation is a good one; the argument should fulfill its standard function in this concrete situation.

Relativity, however, has to be considered here at a different point. The cognitive conditions Lumer formulates - the ability to understand the argument's language, open-mindedness, knowledge about the premises but not the thesis, the ability to grasp the relation between premises and thesis, and so on-have not only to be presupposed on the side of the argument's addressee, but also on the side of the 
evaluator of an argument. That might sound like a triviality at this point, but if we ask for evaluation standards that could be used also in conflicts to decide "objectively" which parties' arguments are better, then all forms of possible biases should be taken into account. Since evaluators of arguments might be more or less able to fulfill the cognitive conditions for themselves that they have to evaluate with regard to an argument's addressee, the argument evaluation itself is relative to the particular constraints and possibilities of an evaluator.

As we will see, this distinction between argument situation, on the one hand, and argument evaluation, on the other, is even more important when it comes to two further forms of relativism in argumentations.

\section{Three forms of relativism}

Our discussion of the cognitive situation of the person an argument is addressing showed that we have to think about more than the "argument itself" when we are looking for evaluation criteria for knowledge promoting argumentations. However, there are two further considerations that force us to go beyond the argument itself. The first one concerns background knowledge that influences the assessment of arguments in a concrete situation, and the second one our general belief-value systems.

The problem of background knowledge in a certain situation becomes visible in one of Lumer's considerations. It is obvious that we need different validity criteria dependent on the type of argument. For what he calls "interpretative argumentation" (also known as "abduction" or "reasoning to the best explanation"), for example, Lumer presents the following "epistemological principle": "a (singular) proposition (about an event) will be true if it is part of the only possible explanation of a known fact" (Lumer 1991, 105). In order to apply this principle, however, certain conditions must be fulfilled. The critical one is that we need to prove "that there is no other set of hypotheses leading to a possible explanation of the explanandum" (105). Such a proof, however, is impossible so that "a valid interpretative argumentation only leads to a probabilist belief", as Lumer says (106).

Applying probability theory, however, is only possible if we have a limited and well-defined set of competing sets of hypotheses. But this is usually the case only in idealized models or in trivial situations. It is hardly the case either in scientific inquiry or in situations in which the perception of a problem is "framed" by constraining background knowledge as, for example, in so-called "intractable" conflicts (cf. Lewicki, Gray, \& Elliott 2003; Hoffmann 2005). In all these situations, it is not only impossible to prove that there are no alternative explanations; it is also impossible to determine the probability of the adequacy of a certain interpretation. In those cases, any evaluation of an argument is limited by the background knowledge an evaluator has at her or his disposal in a concrete situation. 
This knowledge dependency has been emphasized especially by Richard Feldman in his epistemological approach. He argued that:

it is (almost) inevitable that we make argument goodness relative to individuals. This is because background knowledge must play some role in the evaluation of such arguments, and such knowledge obviously varies from one person to another. (Feldman 1994, $182 \mathrm{f}$.)

He gives a nice example. Consider you have to pick up a guest speaker for a sports banquet and you have to decide whether to drive a small or a large car. You know that he is a professional basketball player, so you might formulate the following inductive argument:

(1) Almost all professional basketball players are over six feet tall

(2) The guest speaker is a professional basketball player

(3) The guest speaker is over six feet tall. (Feldman 1994, 177)

This may indeed be a good argument, but what if you would know that the title of the speaker's lecture is "Even Short People Can Make It in the NBA: My Personal Story" (177)? It seems to be obvious that any evaluation of a non-deductive argument depends on the amount of knowledge that is available in a certain situation. And this is not only true with regard to an individual's always limited point of view. Consider the fact that for over 2000 years-from Aristotle to Kant, to be more precise - the theorem that the triangle's inner angles sum up to $180^{\circ}$ has been used by nearly every great philosopher as an example for the possibility that there are "a priori" or "analytical" truths in mathematics. As we know since Lobacevskij (17921856), János Bolyai (1802-1860), and Riemann (1826-1866) however, this theorem is not "absolutely" true; its truth depends on the geometry one chooses to prove it. If Euclidean geometry is the only geometry a civilization knows of, an "argument" that is used to prove this theorem will be a good one while it is a bad one today, at least as long as it does not specify the axiomatic system in which the proof is performed. Since we can never know what kinds of scientific discoveries the future will bring, knowledge relativity remains a principal constraint for argument evaluation.

Dependency on available knowledge, however, is not the only point we should take into account. I would argue that a third form of relativism-besides dependency on the evaluator's cognitive situation and on available background knowledge in a specific situation-concerns the relevance of what can be called the belief-value system of the person who evaluates an argument. Consider the following "real life" argument that was published by the Vatican's Sacred Congregation for the Doctrine of the Faith in 1987:

From the moment of conception, the life of every human being is to be respected in an absolute way because man is the only creature on earth that God has "wished for himself" and the spiritual soul of each man is "immediately created" by God; his whole being bears the image of the Creator. Human life is sacred because from its beginning it involves "the creative 
action of God" and it remains forever in a special relationship with the Creator, who is its sole end. God alone is the Lord of life from its beginning until its end: no one can, in any circumstance, claim for himself the right to destroy directly an innocent human being. (Vatican 1987, Introduction)

This is obviously an "argument" according to the definition I formulated in the introduction to this paper. For the sake of clarity, we might represent it as follows:

(C) "From the moment of conception, the life of every human being is to be respected in an absolute way" (i.e., no one has the right to destroy it) because

$\mathrm{R}_{1}$ it is what God has "wished for himself";

$R_{2}$ its spiritual soul is "immediately created" by God;

$R_{3}$ its whole being bears the image of the Creator;

$\mathrm{R}_{4}$ from its beginning it involves "the creative action of God" and it remains forever in a special relationship with the Creator, who is its sole end; and

$\mathrm{R}_{5}$ God alone is the Lord of life from its beginning until its end.

"Donum Vitae", the "Instruction on Respect for Human Life In its Origin and the Dignity of Procreation: Replies to Certain Questions of the Day", from which this argument is cited, has been formulated as the Vatican's reaction to the then new technologies of in vitro fertilization and embryo transfer. The argument is part of a 2000-year-old history of argumentation, and it is to this day highly influential within public discourse of Western societies, for example with regard to questions like human stem cell research and genetic engineering. But is it a "good" argument or a "bad" one?

I cannot see how any answer to this question could be justified based either on what Biro and Siegel are saying with regard to "the argument itself" or on what Lumer provides as epistemological principles and what Feldman considers as knowledge dependency. Even if Catholics might claim that all the premises of this argument are true and justified so that it would indeed be a question of knowledge that is at stake here, it seems to be more adequate to say that arguments of this sort are based on specific belief-value systems, that is on networks of beliefs (i.e. what is representable in form of a statement concerning a matter of fact) and values (i.e. behavior guiding beliefs that are based on principles, needs, interests, attitudes, etc.) that are consistent from its bearers' point of view. This way, the evaluation of an argument's quality would not only depend on an evaluator's cognitive capacities, and on the background knowledge available in a concrete situation, but also on what Robert C. Pinto called "the broader cognitive community" (Pinto 2001, 135). Pinto already argued that the evaluation of arguments requires us to ask whether it is reasonable to accept an argument "as a member of an historically contingent cognitive community" (133). Obviously, there are arguments whose evaluation will differ depending on social and cultural constraints that are accepted or not accepted within certain communities. 
The problem that makes it necessary, I think, to distinguish between knowledge dependency and dependency on a belief-value system becomes visible when we try to apply the criteria of argument quality developed by Feldman and Goldman. Feldman's final epistemological criterion is the following:

An argument is a good argument for person $S$ if and only if (i) $S$ is justified in believing the conjunction of all the premises of the argument, (ii) $S$ is justified in believing that the premises are "properly connected" to the conclusion, and (iii) the argument is not defeated for $S$. (Feldman 1994, 179)

Goldman, on the other side, who, like Lumer, uses the advancement of knowledge as a criterion to define "conditions" for good practices of argumentative discourse, offers the following four basic conditions:

(1) the speaker believes the asserted conclusion;

(2) the speaker believes each of the asserted premises;

(3) the speaker is justified in believing each of the asserted premises;

(4) the asserted premises jointly provide strong support for the conclusion. (Goldman 1999, 134)

If we consider again our Vatican argument, there is no question that the Sacred Congregation for the Doctrine of the Faith "believes the asserted conclusion" and "each of the asserted premises", and that none of them is "defeated" from the Congregation's point of view. Also Goldman's fourth condition seems to be fulfilled, at least for the Congregation: "the asserted premises jointly provide strong support for the conclusion". If we accept the special relationship between God and the "the life of every human being" as described in all five premises, then we get indeed strong support for the thesis that every human life has to be respected in the way God has to be respected. In this way, as Feldman says, "the premises are 'properly connected' to the conclusion".

The main problem, however, for both these approaches concerns the question of justification. What does it mean that "the speaker" has to be "justified in believing each of the asserted premises"? What kind of "justification" should we expect with regard to any of the premises in our example? There is no question that the Sacred Congregation itself feels justified in believing the premises. This becomes visible in additional footnotes to some of the premises (deleted in my quote) which show that these propositions are themselves part of a longer discussion. Whatever is claimed in this argument is embedded in a larger, consistent belief-value system, and each of the premises can be justified with regard to this system. Or should we expect something like an "objective" justification? In an earlier article, Goldman argued that being "justified in believing" a premise should not only be a "subjective duty", but should be "viewed as (an) independent requirement(s) of objective duty" (1994, 34 f.). That means that "justified beliefs are ones produced by beliefforming processes with high truth ratios; so justified beliefs are likely to be true" (35). 
However, what might be plausible with regard to scientific triths and scientific methods which can be evaluated "by reference to the promotion of true belief and error avoidance" (35) seems to be hopelessly inadequate when it comes to arguments like the one formulated by the Vatican. In his "veritistic epistemology" developed in Knowledge in a Social World, Goldman seems to presuppose that there is only one "truth," since this approach "requires some sort of correspondence theory of truth" (Goldman 1999, 10, $59 \mathrm{ff}$.). But when it comes to genuine conflicts, or what Fogelin (1985) called "deep disagreements", especially those that are based on cultural or religious differences (cf. e.g. Wittes 2005), nobody would disagree, I guess, that there are a lot of so-called "truths" with regard to which it is simply impossible to apply Goldman's "descriptive-success theory" of truth (DS):

An item $X$ (a proposition, a sentence, a belief, etc.) is true if and only if $X$ is descriptively successful, that is, $X$ purports to describe reality and its content fits reality. (Goldman 1999, 59)

Asking whether any of the reasons formulated by the Vatican is "descriptively successful" would move a controversy on this argument only to the next level of fighting for incompatible claims. Based on those observations I would argue that there are many arguments out there to which neither the criterion of being justifiable nor that of promoting truth can be applied successfully. That is, their evaluation does not depend on knowledge claims people can more or less easily agree upon, but on something else that might be called the belief-value system of the person who formulates, or who evaluates, an argument.

\section{Conclusion}

Epistemological approaches to argumentation emphasize that the function of argumentation is not only to convince somebody by any available means, or to reach consensus in conflicts, but also to promote knowledge, that is to generate true or justified beliefs. A goal of epistemologists in argumentation theory is thus to develop and to justify rules and practices of argumentation that "will promote knowledge rather than subvert it" (Goldman 1999, viii; cf. Lumer 1990). For Goldman, for example, argumentation is a "social practice" having a "positive veritistic value", as he calls this property ( $1999,87 \mathrm{ff}$.). His more general goal is to develop a "veritistic epistemology" which is "concerned with the production of knowledge" (1999, 5). "Under veritism we are asked to select the social practices that would best advance the cause of knowledge", that is those practices that generate true beliefs (ibid. 79).

Additionally, there seems to be a certain expectation that if it would be possible to identify those "epistemological principles" and rules of argumentation that are "sufficient conditions for the truth or acceptability of propositions" (Lumer 1991, 101 ), that is, principles that are justifiable as promoting knowledge, then we would get at the same time criteria for the evaluation of arguments which were objective and non-relative in so far as they do not depend on an evaluator's time, place, 
society, culture, historical epoch, conceptual framework, personal training or conviction - as we can define the general meaning of "relativism" following Siegel (1987, xiii). Those criteria were objective and non-relative criteria of argument evaluation, since the only thing an evaluator had to do was to check whether an argumentation follows these rules and principles or not.

The question on which this article focused was whether epistemological approaches to argumentation do indeed provide principles, rules, and standards of argument evaluation that allow us to distinguish clearly between "good" and "bad" arguments. It seems to me without any doubt that the suggestions discussed above allow us to decide at least comparatively which rules and practices of argumentation probably have a more positive impact on the promotion of knowledge than a more negative one. However, the question is whether such a comparative approach is sufficient to allow a non-relative evaluation of argumentations.

We could try, as Lumer suggests with his adequacy principle that demands to check the cognitive situation of an argument's addressee, to include as many factors on which the quality of argumentations depend as possible into the evaluation procedure itself. This way, it would be possible, for example, to evaluate the Vatican's argument with regard to a certain belief-value system that has to be presupposed in order to accept the premises and the conclusion. However, the main problem with such an approach is that the three forms of relativism I distinguished in this paper-dependency on a specific cognitive situation, on available background knowledge, and on a specific belief-value system - are not only relevant for the people who formulate and who are confronted with an argument, but also for the evaluators themselves. Since, as evaluators, we can only take into account what we are aware of, limits of truth are defined by our own individual possibilities and constraints.

\section{References}

Biro, J., \& Siegel, H. (1992). Normativity, Argumentation, and an Epistemic Theory of Fallacies. In F. H. v. Eemeren, R. Grootendorst, J. A. Blair \& C. A. Williard (Eds.), Argumentation Illuminated: Selected Papers from the 1990 International Conference on Argumentation (pp. 81-103). Dordrecht: Foris.

Boger, G. (2005). Subordinating Truth-Is Acceptability Acceptable? Argumentation, $19(2), 187-238$.

Feldman, R. (1994). Good Arguments. In F. F. Schmitt (Ed.), Socializing epistemology The social dimensions of knowledge (pp. 159-18.8). Lanham, MD: Rowman \& Littlefield Publishers.

Fogelin, R. (1985). The Logic of Deep Disagreements. Informal Logic, 7, 1-8.

Goldman, A. I. (1994). Argumentation and Social Epistemology. The Journal of Philosophy, 9/(1), 27-49. 
Goldman, A. I. (1999). Knowledge in a Social World. Oxford: Clarendon Press.

Harding, S. (1996 <1993>). Rethinking standpoint epistemology: What is 'strong objectivity'? In E. F. Keller \& H. E. Longino (Eds.), Feminism and science (pp. 235248). Oxford/New York: Oxford University Press.

Hoffmann, M. H. G. (2005). Logical argument mapping: A method for overcoming cognitive problems of conflict management. International Journal of Conflict Management, 16(4), 305-335.

Horkheimer, M., \& Adorno, T. W. (2002 <1947>). Dialectic of Enlightenment. Philosophical Fragments (Dialektik der Auffklärung) (E. Jephcott, Trans.). Stanford, Calif:: Stanford University Press.

Lewicki, R. J., Gray, B., \& Elliott, M. (Eds.). (2003). Making Sense of Intractable Environmental Conflicts. Concepts and Cases. Washington - Covelo - London: Island Press.

Longino, H. E. (1996 <1993>). Subjects, Power, and Knowledge: Description and Prescription in Feminist Philosophies of Science. In E. F. Keller \& H. E. Longino (Eds.), Feminism and science (pp. 264-279). Oxford; New York: Oxford University Press.

Lumer, C. (1990). Praktische Argumentationstheorie: Theoretische Grundlagen, Praktische Begriundung und Regeln Wichtiger Argumentationsarten. Braunschweig; Wiesbaden: Vieweg.

Lumer, C. (1991). Structure and Function of Argumentations. An Epistemological Approach to Determining Criteria for the Validity and Adequacy of Argumentations. In F. H. v. Eemeren (Ed.), Proceedings of the Second International Conference on Argumentation. Organized by the International Society for the Study of Argumentation (ISSA) at the University of Amsterdam, June 19-22, 1990 (pp. 98107). Amsterdam: Sicsat.

Lyotard, J. F. (1984 <1979>). The Postmodern Condition. A Report on Knowledge (G. Bennington \& B. Massumi, Trans.). Minneapolis University of Minnesota Press.

Lyotard, J. F. (1993). Toward the postmodern. Atlantic Highlands, N.J.: Humanities Press International.

Pinto, R. C. (2001). Argument, Inference and Dialectic. Collected Papers on Informal Logic. Dordrecht; Boston: Kluwer Academic.

Siegel, H. (1987). Relativism Refuted. Dordrecht: Reidel

Siegel, H. (1999). Argument Quality and Cultural Difference. Argumentation, I3(2), 183 201.

Siegel, H., \& Biro, J. (1997). Epistemic Normativity, Argumentation, and Fallacies. Argumentation, II(3), 277-292.

Smith, D. E. (1990). The Conceptual Practices of Power: A Feminist Sociology of Knowledge. Boston: Northeastern University Press.

Vatican (1987). The Gift of Life (Donum Vitae). Instruction on Respect for Human Life In its Origin and the Dignity of Procreation: Replies to Certain Questions of the Day. http://www. nccbuscc. org/prolife/tdocs/donumvitae. htm.

Vattimo, G. $(1991<1985>)$. The End of Modernity. Nihilism and Hermeneutics in Postmodern Culture: The Johns Hopkins University Press. 
Williams, B. (2002). Truth \& Truthfulness: An Essay in Genealogy: Princeton Univ. Pr.

Wittes, T. C. (Ed.). (2005). How Israelis and Palestinians Negotiate: A Cross Cultural Analysis of the Oslo Peace Process. Washington: United States Institute of Peace Press.

Michael H.G. Hoffmann

School of Public Policy

Georgia Institute of Technology

D.M. Smith Building 685 Cherry Street

Atlanta, GA 30332-0345

michael.hoffmann@pubpolicy.gatech.edu 